\title{
Supplementary Online Materials
}

Polygenic score for educational attainment captures DNA variants shared between personality traits and educational achievement

\section{Authors:}

Emily Smith-Woolley ${ }^{1 *}$, Saskia Selzam ${ }^{1 *}$, Robert Plomin ${ }^{1}$

*Joint first authors

Supplementary Methods 2

Methods S1 - Structural equation model of personality domains, GCSE results and polygenic scores .......2

Supplementary Tables 3

Table S1 - Descriptive statistics of all variables for the full sample 3

Table S2 - Sensitivity analysis of missingness of personality/motivation composites on socio-economic status, general cognitive ability, and GCSE grades .5

Table S3 - Descriptive statistics of all variables for the sample of unrelated individuals .6

Supplementary Figures

Figure S1 - Correlations across all individual measures of personality and motivation, the personality/motivation composites and polygenic scores 7

Figure S2 - Results from the parallel analysis .8

Figure S3 - Correlations across all polygenic scores and personality/motivation domains. 9

Figure S4 - Correlations across all EduYears GPS thresholds and personality/motivation domains 10

Figure S5 - Correlations across all Neuroticism GPS thresholds and personality/motivation domains.......11

Figure S6 - Correlations across all Wellbeing GPS thresholds and personality/motivation domains..... 12

Figure S7 - Correlations between the 2016 EduYears GPS and outcome measures .12 


\section{Supplementary Methods}

Methods S1 - Structural equation model of personality domains, GCSE results and polygenic scores

To test the extent to which the covariance between personality domains and GCSE results are explained by the polygenic scores, we used structural equation modelling. Because we assume causality from polygenic score (an aggregate score of DNA variants) to outcome variables, we applied the following model to our z-standardized variables:

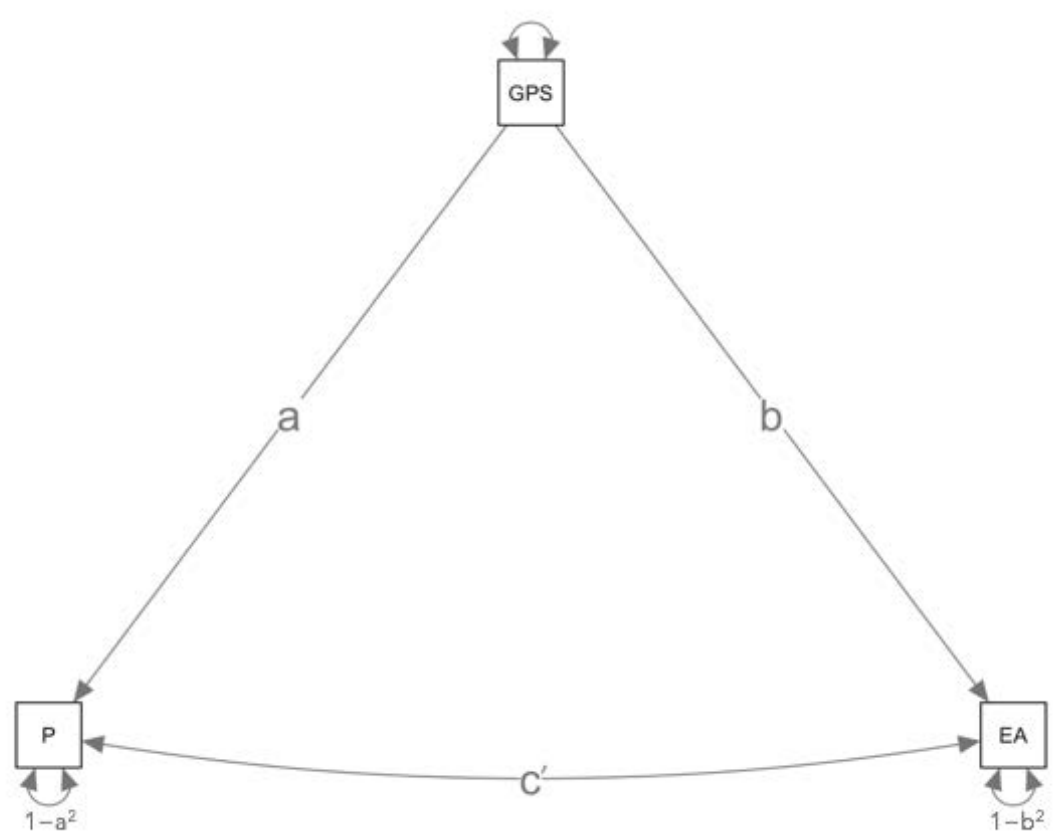

Note. $\mathrm{P}=$ personality trait, EA = educational achievement defined as GCSE results, GPS = genome-wide polygenic score

Paths $a, b$ and $c$ are the beta effect size parameters. The GPS effect is described by the product of $a$ and $b$, which is the pathway from the causal variable GPS to P, and GPS to EA. Path c' describes the residual relation between $P$ and EA after accounting for the effects of the causal variable GPS in $P$ and EA, respectively. The total effect can be derived by summing the effects of the residual and the indirect path, described as c' + ab. To calculate the proportion of the total effect that is explained by the causal variable GPS, the effect of the indirect path ab is divided by the total effect c' $+a b$. 


\section{Supplementary Tables}

Table S1 - Descriptive statistics of all variables for the full sample

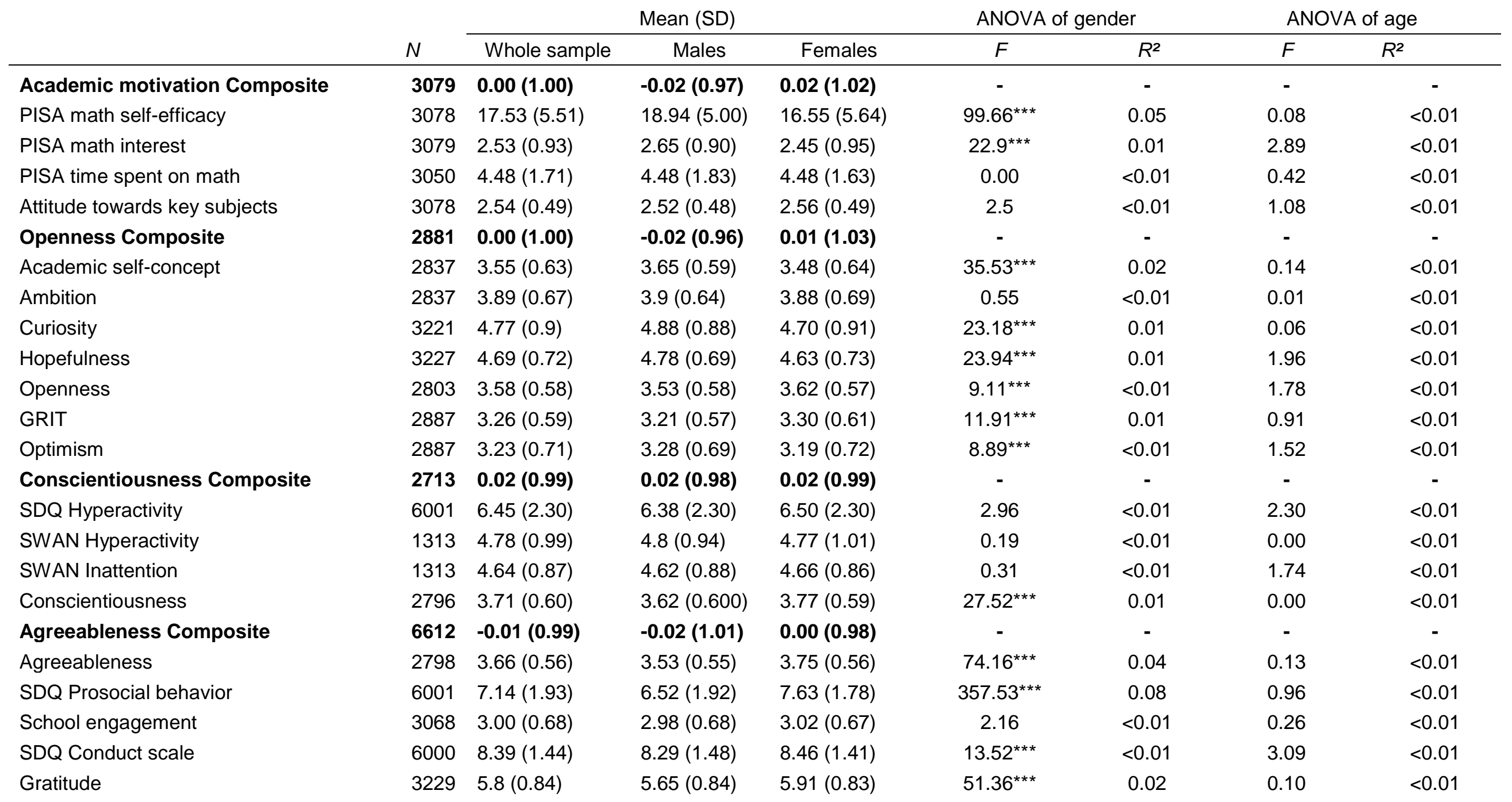




\section{Neuroticism Composite}

Cognitive Disorganization

CASI anxiety

MFQ

Subjective happiness

Life satisfaction

Peer problems

Neuroticism

\section{Extraversion}

GCSE

general cognitive ability

\section{EduYears GPS}

Neuroticism GPS

Wellbeing GPS

$\begin{array}{rlll}\mathbf{6 0 0 8} & -\mathbf{0 . 0 1 ( 1 . 0 1 )} & -\mathbf{0 . 0 2 ( 0 . 9 0 )} & \mathbf{0 . 0 0 ( 1 . 0 8 )} \\ 5998 & 3.93(2.86) & 3.37(2.73) & 4.38(2.88) \\ 6004 & 8.07(5.96) & 6.20(4.85) & 9.53(6.34) \\ 6003 & 3.59(4.33) & 2.56(3.29) & 4.39(4.85) \\ 5998 & 2.88(0.94) & 2.89(0.91) & 2.87(0.97) \\ 3224 & 2.40(0.62) & 2.38(0.58) & 2.41(0.64) \\ 6001 & 1.54(1.51) & 1.60(1.53) & 1.48(1.49) \\ 2808 & 2.58(0.68) & 2.47(0.64) & 2.66(0.69) \\ \mathbf{2 8 0 7} & \mathbf{0 . 0 0 ( 0 . 9 7 )} & -\mathbf{0 . 0 1 ( 0 . 9 7 )} & \mathbf{0 . 0 1 ( 0 . 9 7 )} \\ \mathbf{8 3 2 2} & -\mathbf{0 . 0 1 ( 1 . 0 0 )} & -\mathbf{0 . 0 2 ( 1 . 0 2 )} & \mathbf{0 . 0 0 ( 0 . 9 9 )} \\ \mathbf{3 9 3 9} & \mathbf{0 . 0 1 ( 0 . 9 9 )} & \mathbf{0 . 0 0 ( 0 . 9 8 )} & \mathbf{0 . 0 1 ( 0 . 9 9 )} \\ \mathbf{1 0 3 4 6} & \mathbf{0 . 0 1 ( 1 . 0 0 )} & \mathbf{0 . 0 2 ( 1 . 0 1 )} & \mathbf{0 . 0 0 ( 0 . 9 9 )} \\ \mathbf{1 0 3 4 6} & \mathbf{0 . 0 1 ( 1 . 0 0 )} & \mathbf{0 . 0 1 ( 1 . 0 1 )} & \mathbf{0 . 0 1 ( 0 . 9 9 )} \\ \mathbf{1 0 3 4 6} & \mathbf{0 . 0 0 ( 1 . 0 0 )} & \mathbf{0 . 0 0 ( 0 . 9 9 )} & \mathbf{0 . 0 0 ( 1 . 0 1 )}\end{array}$

-
$126.06^{\star \star \star}$
$332.69^{\star \star \star}$
$183.36^{\star \star \star}$
0.18
1.76
$6.61^{\star}$
$35.82^{\star \star \star}$
$\mathbf{0 . 3 2}$
$\mathbf{0 . 7 9}$
-
$\mathbf{1 . 0 8}$
$\mathbf{0 . 0 2}$
$\mathbf{0 . 0 0}$

0.03
0.08
0.04
$<0.01$
$<0.01$
$<0.01$
0.02
$<0.01$
$<0.01$
-
$<0.01$
$<0.01$
$<0.01$

$<0.01$

$<0.01$

$<0.01$

$<0.01$

$<0.01$

$<0.01$

$<0.01$

$<0.01$

$<0.01$

$<0.01$

$<0.01$

Note: Means and standard deviations for individual measures are calculated based on raw data. Means and standard deviations for domains are calculated with z-standardised age and sex regressed data. Values of standard deviation are given in parentheses. ${ }^{+}=$standardization of the individual cognitive scales assessed at age 7,12 and 16 was required to form this composite. $\mathrm{N}=$ sample size after exclusions. For DZ twin pairs, ANOVA performed on one randomly selected twin per pair to test the effect of sex and age. Results $=F$ statistic; ${ }^{*}=p<.05 ;{ }^{* *}=p<.01 ; R^{2}=p r o p o r t i o n$ of variance explained. 
Table S2 - Sensitivity analysis of missingness of personality/motivation composites on socio-economic status, general cognitive ability, and GCSE grades

\begin{tabular}{|c|c|c|c|c|c|}
\hline \multicolumn{6}{|c|}{ GCSE } \\
\hline & $\begin{array}{c}\text { Present } \\
\text { M (SD) }\end{array}$ & $\begin{array}{c}\text { Missing } \\
\text { M (SD) }\end{array}$ & $\boldsymbol{z}$ & $p$ & $R^{2}$ \\
\hline Academic motivation & $0.14(0.97)$ & $-0.09(1.01)$ & 8.01 & $6.63 \mathrm{E}-16$ & 0.009 \\
\hline Openness Composite & $0.15(0.96)$ & $-0.09(1.01)$ & 8.46 & $1.31 \mathrm{E}-17$ & 0.010 \\
\hline Conscientiousness Composite & $0.17(0.98)$ & $-0.09(1.00)$ & 8.69 & $1.73 E-18$ & 0.011 \\
\hline Agreeableness Composite & $0.10(0.98)$ & $-0.30(1.01)$ & 12.73 & 5.77E-39 & 0.026 \\
\hline Neuroticism Composite & $0.12(0.98)$ & $-0.26(1.00)$ & 13.07 & 6.06E-41 & 0.025 \\
\hline Extraversion & $0.17(0.95)$ & $-0.09(1.01)$ & 9.23 & $1.00 \mathrm{E}-20$ & 0.013 \\
\hline \multicolumn{6}{|c|}{ General cognitive ability } \\
\hline & $\begin{array}{l}\text { Present } \\
\text { M (SD) }\end{array}$ & $\begin{array}{c}\text { Missing } \\
\text { M (SD) }\end{array}$ & $\boldsymbol{z}$ & $p$ & $R^{2}$ \\
\hline Academic motivation & $0.03(0.98)$ & $-0.05(0.99)$ & 1.96 & 0.05 & 0.001 \\
\hline Openness Composite & $0.03(0.99)$ & $-0.05(0.99)$ & 2.09 & 0.04 & 0.001 \\
\hline Conscientiousness Composite & $0.09(0.97)$ & $-0.11(1.00)$ & 5.16 & $1.98 \mathrm{E}-07$ & 0.007 \\
\hline Agreeableness Composite & $0.03(0.98)$ & $-0.16(1.03)$ & 3.22 & $1.24 \mathrm{E}-3$ & 0.005 \\
\hline Neuroticism Composite & $0.06(0.98)$ & $-0.17(1.00)$ & 5.26 & 1.18E-07 & 0.009 \\
\hline Extraversion & $0.05(0.97)$ & $-0.08(1.01)$ & 3.41 & $6.16 \mathrm{E}-4$ & 0.003 \\
\hline \multicolumn{6}{|c|}{ SES } \\
\hline & $\begin{array}{c}\text { Present } \\
\text { M (SD) }\end{array}$ & $\begin{array}{c}\text { Missing } \\
\text { M (SD) }\end{array}$ & $\boldsymbol{Z}$ & $p$ & $R^{2}$ \\
\hline Academic motivation & $0.2(0.98)$ & $0.04(1.01)$ & 5.91 & 3.09E-09 & 0.004 \\
\hline Openness Composite & $0.21(0.98)$ & $0.04(1.00)$ & 6.23 & $3.96 \mathrm{E}-10$ & 0.005 \\
\hline Conscientiousness Composite & $0.22(0.98)$ & $0.04(1.00)$ & 6.26 & $3.20 \mathrm{E}-10$ & 0.005 \\
\hline Agreeableness Composite & $0.21(0.98)$ & $-0.14(1.00)$ & 13.47 & $7.88 \mathrm{E}-43$ & 0.022 \\
\hline Neuroticism Composite & $0.23(0.98)$ & $-0.12(1.00)$ & 13.85 & $3.02 E-45$ & 0.022 \\
\hline Extraversion & $0.22(0.98)$ & $0.04(1.01)$ & 6.67 & 2.07E-11 & 0.006 \\
\hline
\end{tabular}

Note: SES = socio-economic status; GCSE = General Certificate for Secondary Education; present = data is nonmissing for the respective composite; missing $=$ data is missing for the respective composite. Analyses were performed on unrelated individuals only, where one twin per twin pair was randomly selected if data was available on dizygotic twin pairs. Degrees of freedom (df) for GCSE analyses = 5,600; df for general cognitive ability analyses = 2,$676 ;$ df for SES analyses $=6,569$. 
POLYGENIC SCORE EXPLAINS SHARED VARIANCE BETWEEN PERSONALITY TRAITS AND

EDUCATIONAL ACHIEVEMENT

Table S3 - Descriptive statistics of all variables for the sample of unrelated individuals

\begin{tabular}{lcccccc} 
& $N$ & Mean & SD & Skew & Min & Max \\
\hline Academic motivation Composite & 2084 & 0.00 & 1.00 & -0.57 & -4.4 & 2.49 \\
Openness Composite & 1958 & 0.00 & 1.00 & -0.21 & -4.48 & 3.12 \\
Conscientiousness Composite & 1838 & 0.02 & 0.99 & -0.24 & -3.52 & 2.6 \\
Agreeableness Composite & 4415 & -0.01 & 0.99 & -0.62 & -4.56 & 2.62 \\
Neuroticism Composite & 4005 & -0.01 & 1.01 & 0.89 & -2.14 & 5.37 \\
Extraversion & 1909 & 0.00 & 0.97 & -0.42 & -3.86 & 2.26 \\
GCSE & 5602 & -0.01 & 1.00 & -0.43 & -3.63 & 1.87 \\
EduYears GPS & 7026 & 0.01 & 1.00 & 0.08 & -3.49 & 3.71 \\
Neuroticism GPS & 7026 & 0.01 & 1.00 & 0.04 & -5.39 & 4.34 \\
Wellbeing GPS & 7026 & 0.00 & 1.00 & 0.01 & -3.96 & 3.78 \\
\hline
\end{tabular}




\section{Supplementary Figures}

Figure S1 - Correlations across all individual measures of personality and motivation, the personality/motivation composites and polygenic scores

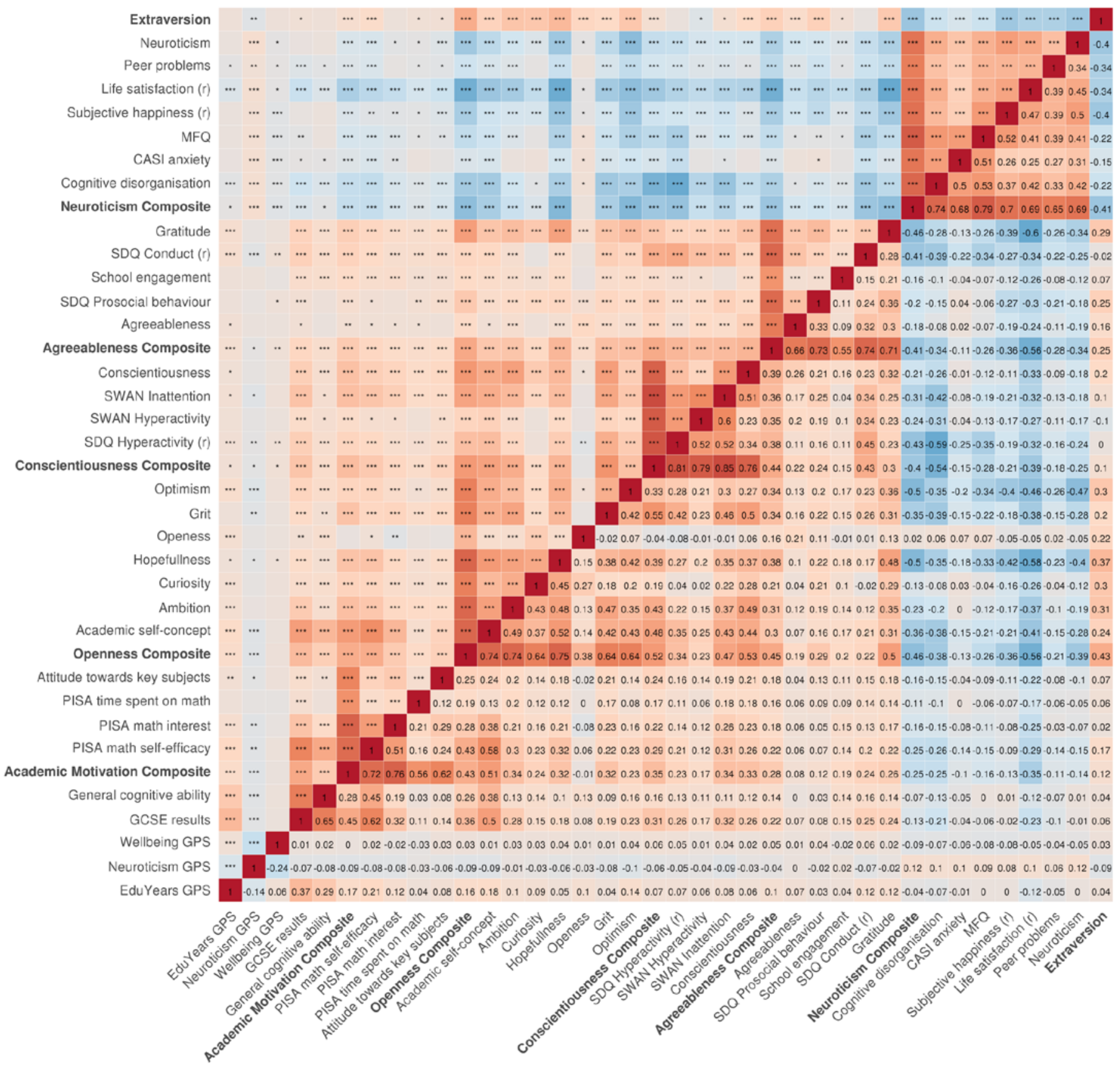

Note: $(\mathrm{r})=$. recoded so that higher scores were positive, i.e. less conduct problems. Variable labels in bold represent composites made up of the succeeding individual scales. ${ }^{*}=p<0.05$; ${ }^{* \star}=p<0.001 ;{ }^{* \star}=p<$ 0.0001. 
Figure S2 - Results from the parallel analysis.

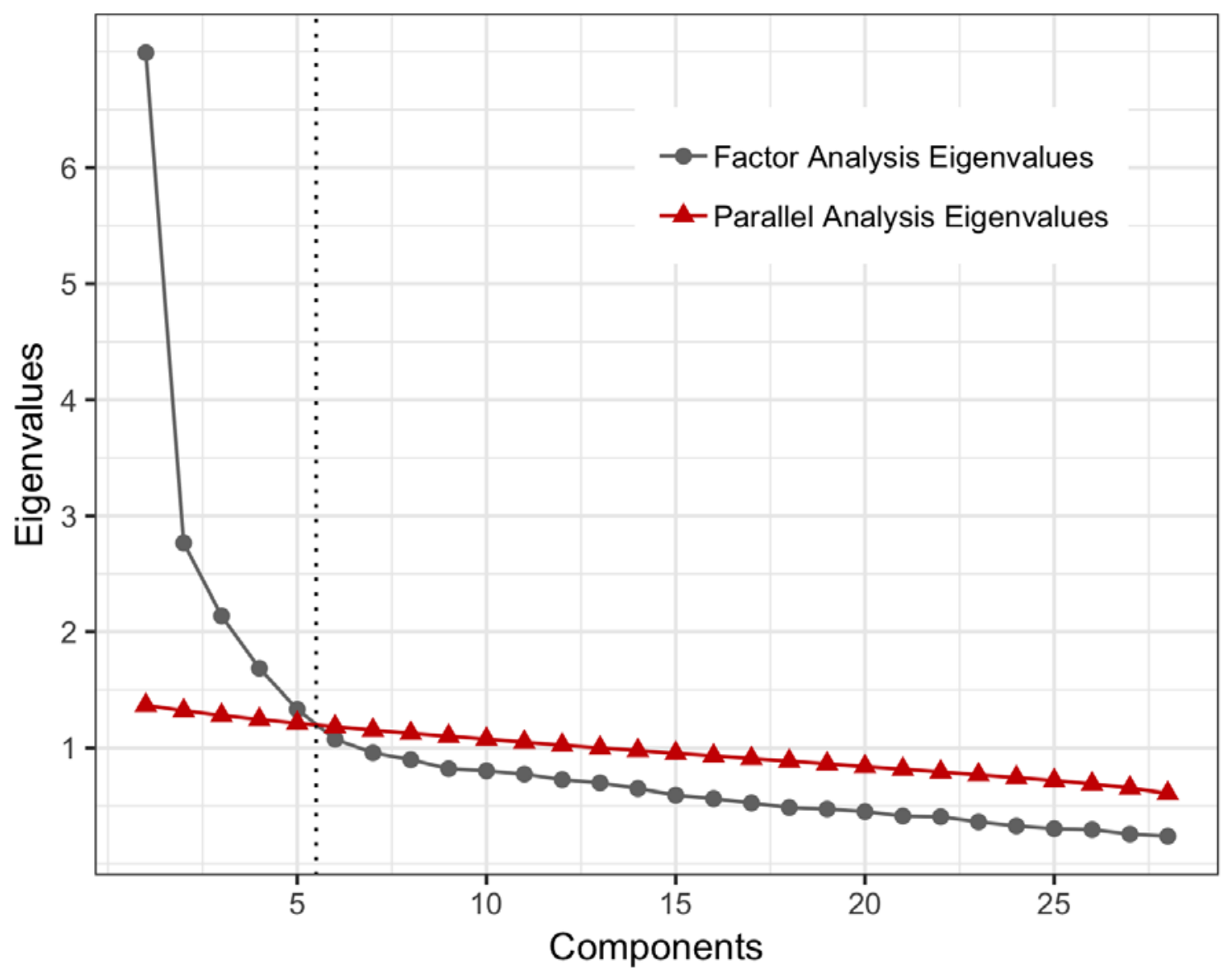

Note: The dotted line represents the point at which the factor eigenvalue in the study becomes smaller than the $95^{\text {th }}$ percentile of the simulated eigenvalues from parallel analysis 
Figure S3 - Correlations across all polygenic scores and personality/motivation domains

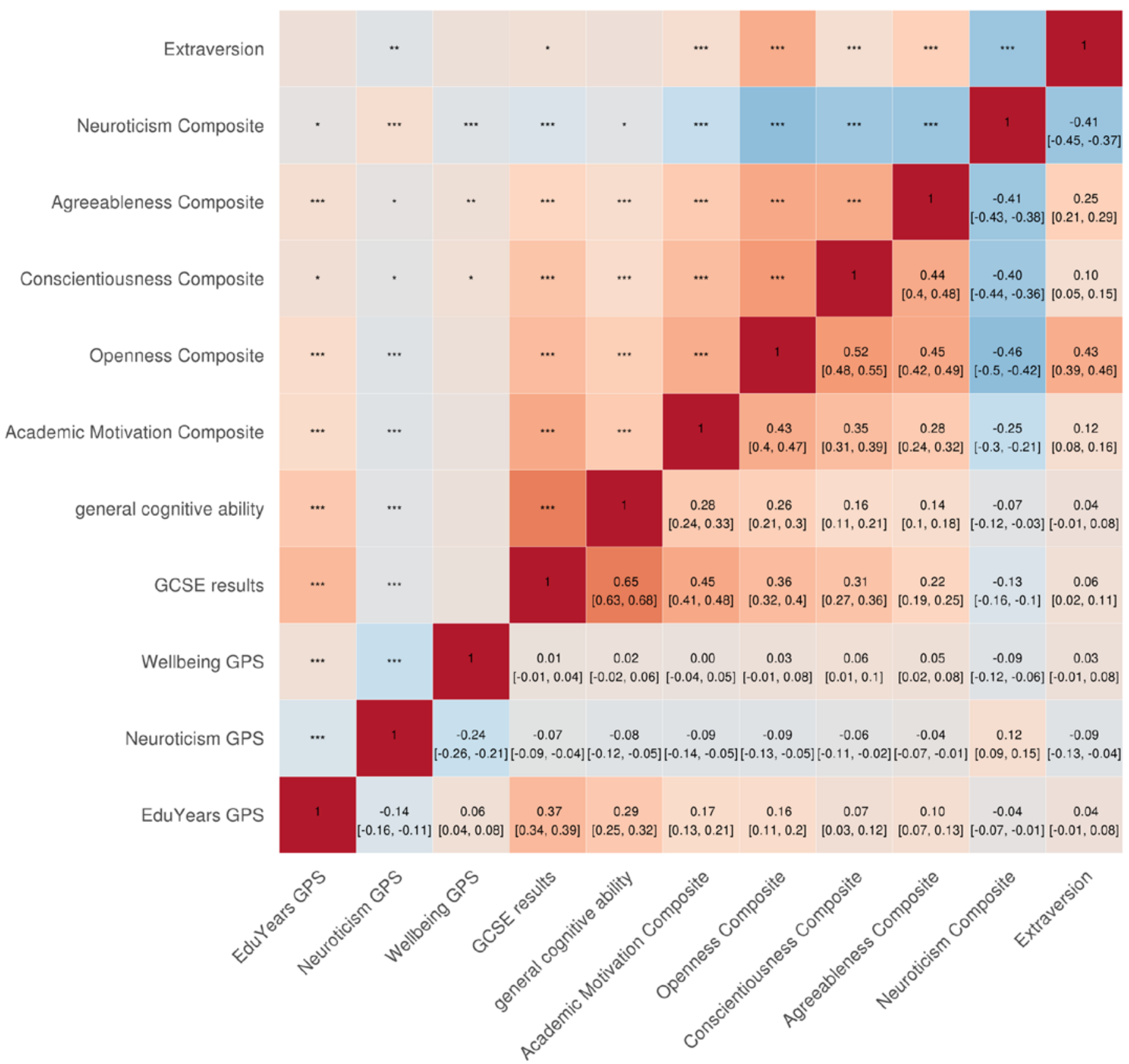

Note: 95\% confidence intervals of the Pearson's correlation coefficients shown in square brackets. ${ }^{*}=p<$ $0.05 ; * \star=p<0.001 ; * \star *=p<0.0001$. 
Figure S4 - Correlations across all EduYears GPS thresholds and personality/motivation domains

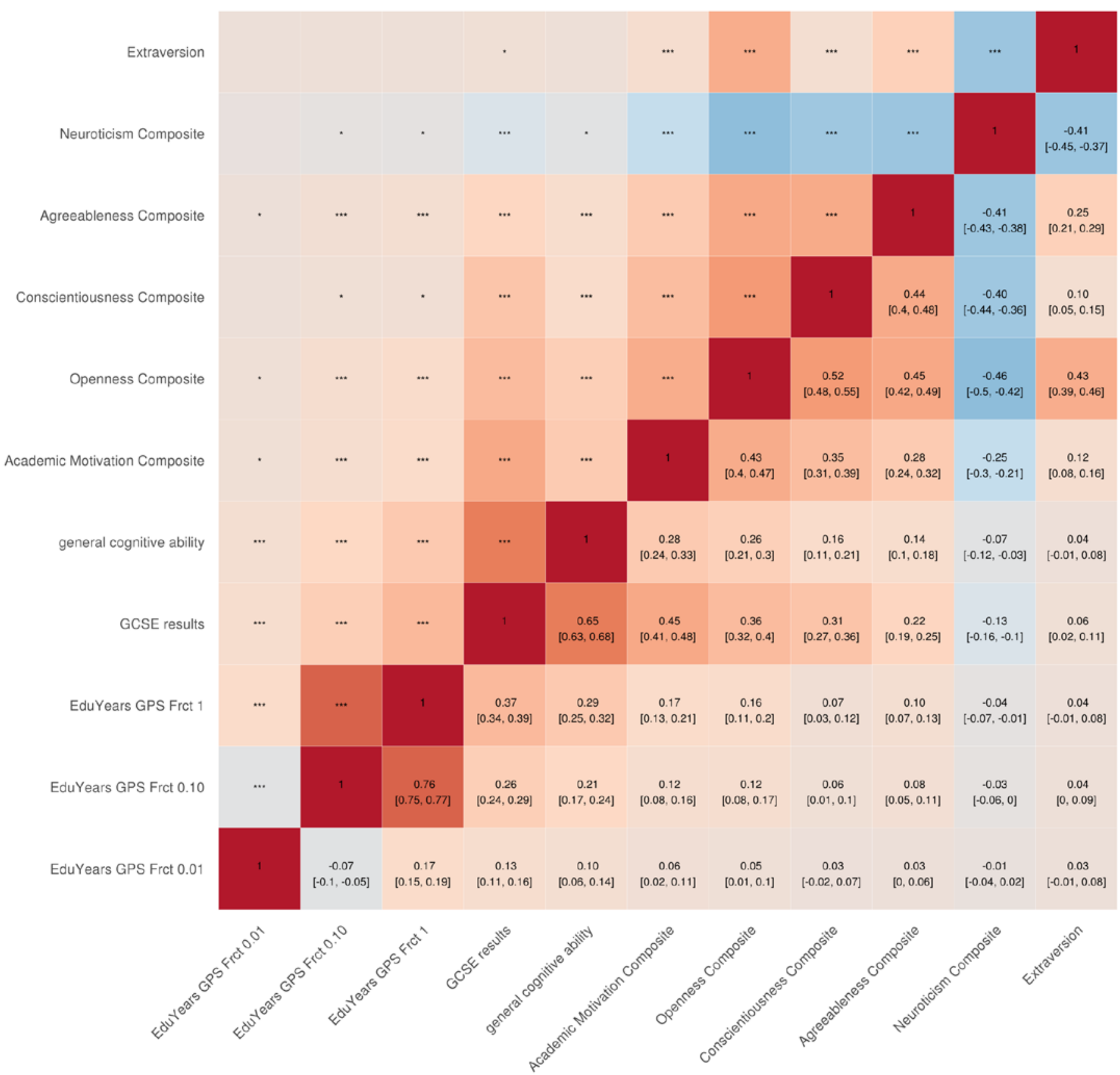

Note: $95 \%$ confidence intervals of the Pearson's correlation coefficients shown in square brackets. ${ }^{*}=p<$ $0.05 ;{ }^{* *}=\mathrm{p}<0.001 ;{ }^{* *}=\mathrm{p}<0.0001$. 
Figure S5 - Correlations across all Neuroticism GPS thresholds and personality/motivation domains

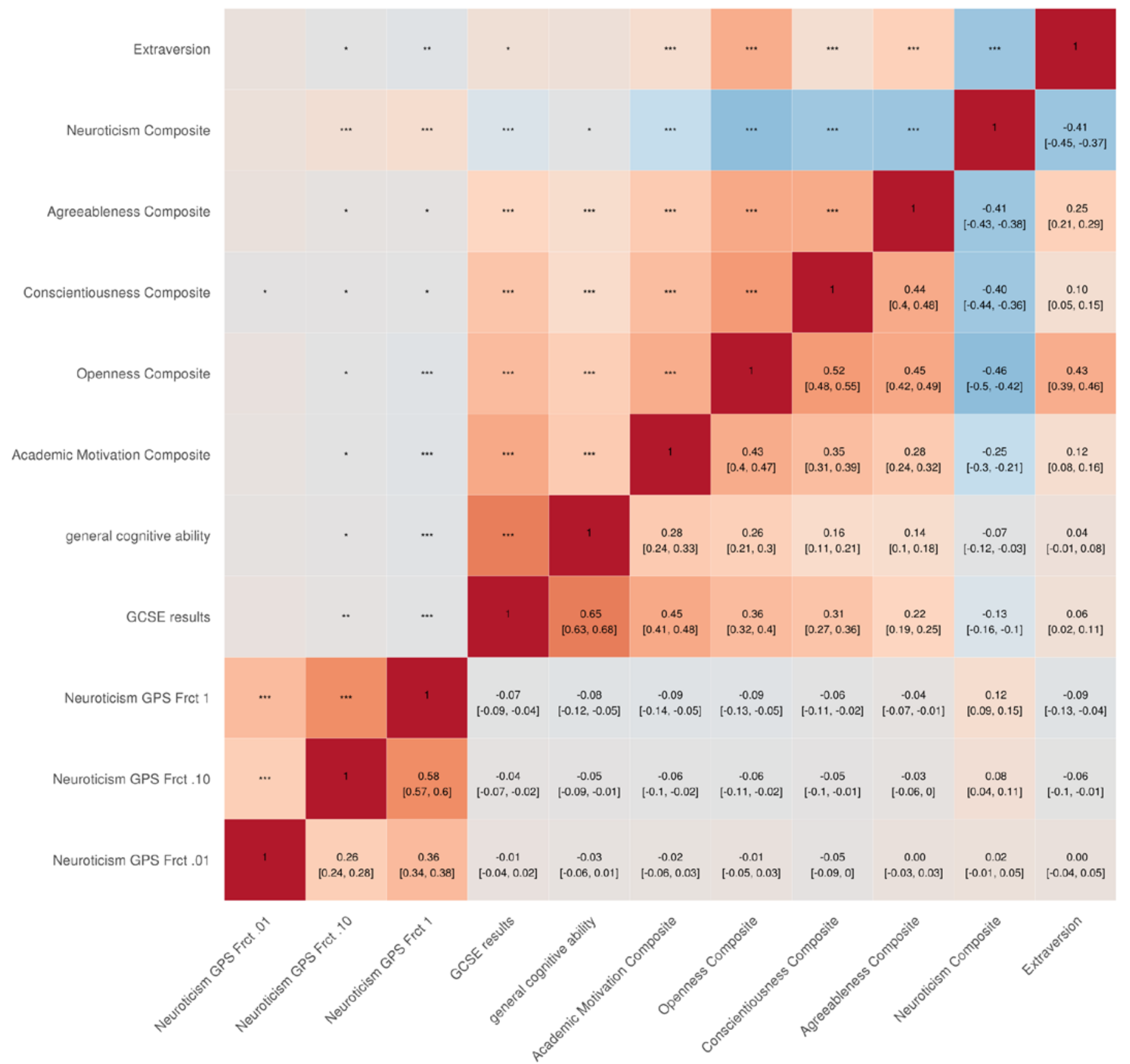

Note: $95 \%$ confidence intervals of the Pearson's correlation coefficients shown in square brackets. ${ }^{*}=p<$ $0.05 ;{ }^{* *}=p<0.001 ;{ }^{* \star *}=p<0.0001$. 
Figure S6 - Correlations across all Wellbeing GPS thresholds and personality/motivation domains

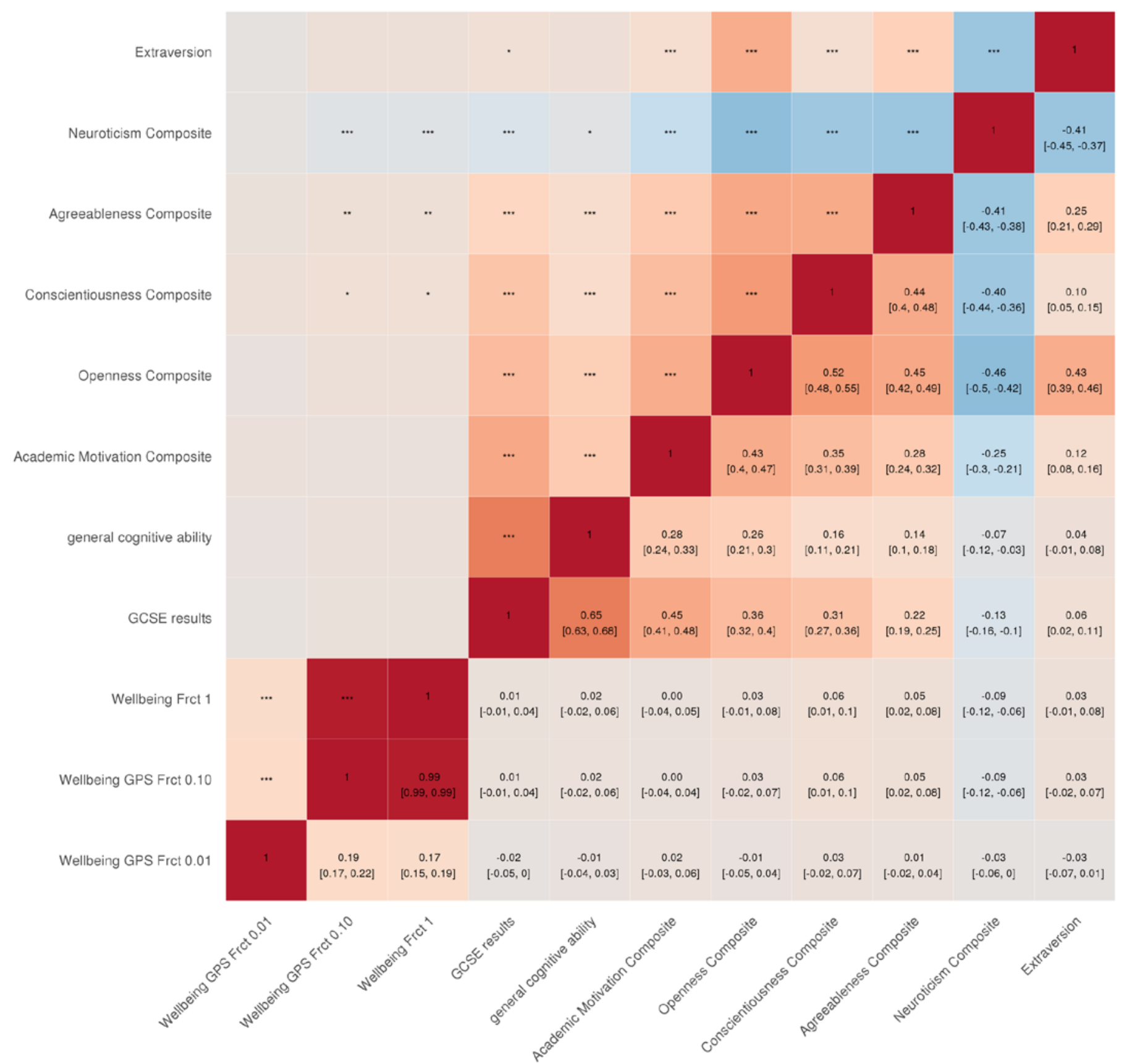

Note: $95 \%$ confidence intervals of the Pearson's correlation coefficients shown in square brackets. ${ }^{*}=p<$ $0.05 ;{ }^{* \star}=\mathrm{p}<0.001 ;{ }^{* \star *}=\mathrm{p}<0.0001$. 
Figure S7 - Correlations between the 2016 EduYears GPS and outcome measures

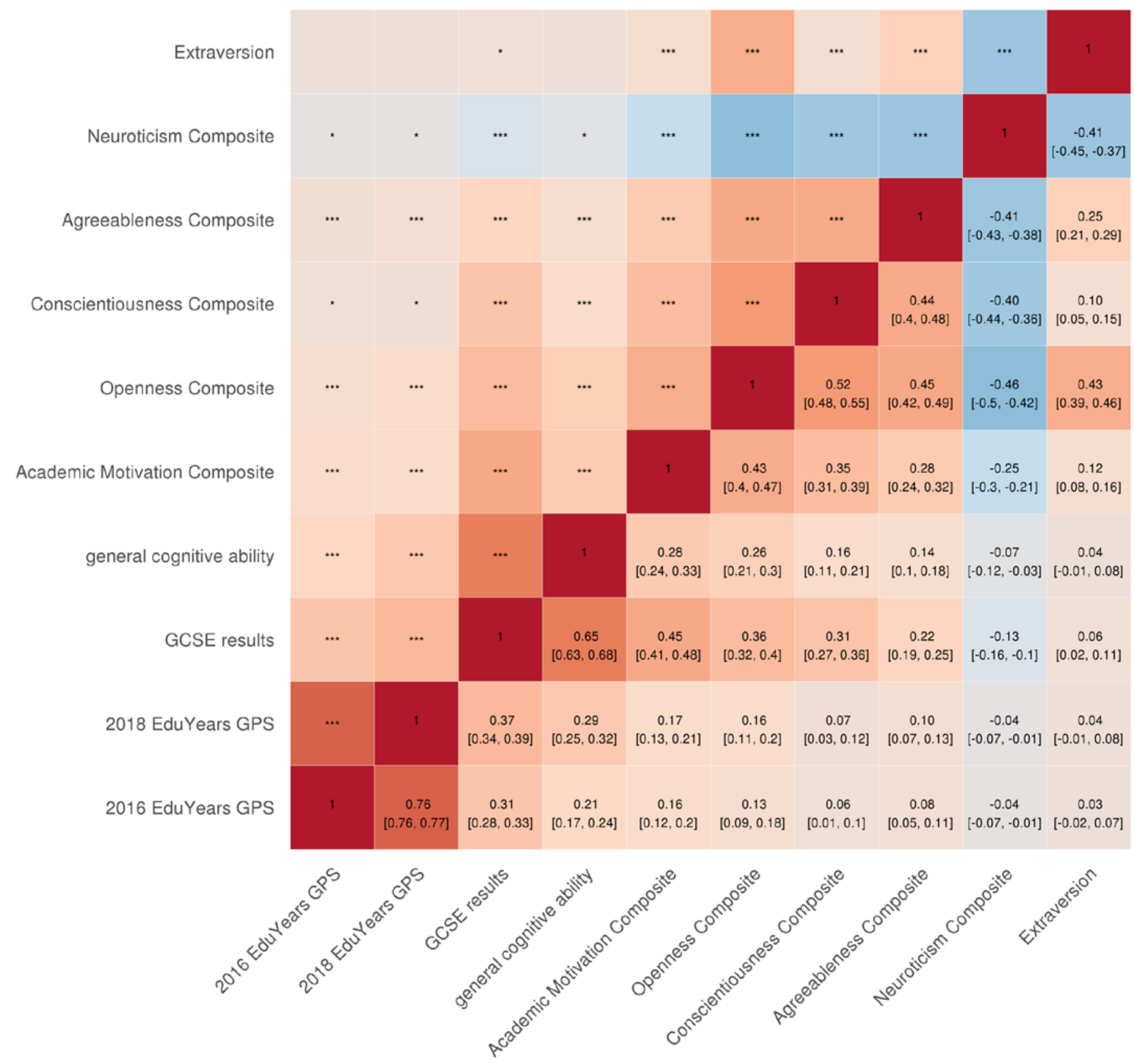

Note: 95\% confidence intervals of the Pearson's correlation coefficients shown in square brackets. ${ }^{*}=p<$ $0.05 ;{ }^{* *}=\mathrm{p}<0.001 ;{ }^{* * *}=\mathrm{p}<0.0001$. 